\title{
Social anxiety in E-Learning: Scale validation and socio- demographic correlation study
}

\author{
Ali S Alsudais ${ }^{1,2} \cdot$ Abdullah S Alghamdi $^{1,2} \cdot$ Abdullrhman A Alharbi $^{1,2}$. \\ Atif A Alshehri ${ }^{1,2} \cdot$ Mustafa A Alzhrani ${ }^{1,2} \cdot$ Sinan Keskin ${ }^{3} \cdot$ Muhittin Şahin ${ }^{4}$. \\ Alaa M Althubaiti ${ }^{1,2,5}$ (D)
}

Received: 19 July 2021 / Accepted: 26 January 2022 / Published online: 3 March 2022

(c) The Author(s), under exclusive licence to Springer Science+Business Media, LLC, part of Springer Nature 2022

\begin{abstract}
During the ongoing coronavirus disease 2019 pandemic, over 1.5 billion students worldwide have been deprived of access to traditional learning. This situation has necessitated the use of social distancing-based educational methods; consequently, a tremendous shift towards e-learning has been observed. This study assesses medical students' social anxiety levels in e-learning environments. The study was conducted in two phases. In the first phase, the original Turkish Social Anxiety Scale for E-Learning Environments (SASE) was adapted in English and tested for validity and reliability. This instrument has two subscales: social anxiety in learner-learner interaction and in learner-instructor interaction. In the second stage, we explored the associations of gender, age, and perceived academic performance with medical students' social anxiety levels in e-learning environments. A total of 325 responses were analysed. Consistent with the original version, the adapted scale is a reliable and valid measure of social anxiety in e-learning. Social anxiety in e-learning was related to gender $(p=0.008)$ and age $(p=0.013)$. Social anxiety levels were higher in students with lower perceived performance during e-learning compared to students with enhanced performance, but the difference was not significant. The SASE is a useful instrument for evaluating social anxiety in e-learning environments across English educational frameworks. Considering the shift in social interaction environments, efforts are required to reduce medical students' social anxiety levels and enhance learning.
\end{abstract}

Keywords Social anxiety $\cdot$ E-learning $\cdot$ Confirmatory factor analysis $\cdot$ Medical students

Extended author information available on the last page of the article 


\section{Introduction}

Social constructivist learning theory posits that people construct knowledge through social and communal interactions (Van Ameringen et al., 2003). Many people do not have the motivation to pursue academic life owing to social and cooperative concerns, such as social anxiety. Social anxiety in adults is the most prevalent form of anxiety (Bandelow \& Michaelis, 2015; Neufeld et al., 2020). It is best described as sustained fear and shyness during social interactions (Stein \& Stein, 2008). In unfamiliar social gatherings, individuals with social anxiety tend to be quiet, interact less actively, and avoid speaking and expressing their perspectives. Low self-esteem and high selfcriticism have been shown to be linked to social anxiety (Cox et al., 2004). Owing to its pernicious onset and clinical manifestation, social anxiety is associated with difficulty in concentration, low educational achievement, and reduced professional accomplishments in unfamiliar social settings (Stein \& Stein, 2008; Al-Hazmi et al., 2020). Further, social anxiety is linked to several comorbidities, including major depression disorder, other anxiety disorders, and substance abuse (Leichsenring \& Leweke, 2017).

Several studies have been conducted to evaluate social anxiety among university students (Al-Hazmi et al., 2020; Hakami et al., 2018; Reta et al., 2020). One study in the context of traditional learning environments revealed that more than $50 \%$ of students experienced social anxiety in their academic life (Al-Hazmi et al., 2020). Symptom severity was negatively correlated with grade point average (GPA), academic performance, as well as social and clinical skills. The suggested risk factors for social anxiety were age, marital status, monthly income, and academic level.

In this technological era, most institutions provide online programmes to facilitate learning. In e-learning, technology serves as the basis for the dissemination of knowledge (Vaona et al., 2018). This includes the use of electronic devices, the internet, and programmes that can provide the necessary, adequately flexible tools for use in learning sessions.

Social anxiety has always been a concern in traditional education, but it has never been examined in e-learning environments. During the ongoing coronavirus disease 2019 pandemic, over 1.5 billion students worldwide have had their access to traditional learning compromised (UNESCO, 2020), creating a necessity for an alternative method; a worldwide shift to online-based education has been the answer. The goal of this study was to establish a scale to measure social anxiety in e-learning environments and to examine how this anxiety correlates with students' socio-demographic characteristics.

\section{Method}

This research was conducted in two stages to investigate the social anxiety levels of medical students. In the first phase, the original Turkish version of the Social Anxiety Scale for E-Learning Environments (SASE) was adapted in English. In the second stage, the associations between gender, age, perceived academic performance during e-learning, and social anxiety levels of medical students in e-learning environments 
were explored. This phase was conducted during the academic year 2020-2021 with undergraduate medical students at the College of Medicine, King Saud bin Abdulaziz University for Health Sciences, Jeddah, Saudi Arabia. The study targeted a total of 781 male and female undergraduate medical students from 3rd to 6th year considering that the university's medicine bachelor programme commences after two years of a pre-professional programme. During the ongoing pandemic, the university adopted a common approach to fulfil the e-learning process utilising different platforms such as Blackboard Collaborate and Microsoft Teams. Through these platforms, students could attend, participate, present, discuss, and meet with each other to accomplish the required educational tasks such as problem-based learning sessions, community and doctor theme presentations, personal and professional development presentations, as well as course lectures. Additionally, video-based sessions were conducted to substitute the required physical presence during practical lectures. Data was collected approximately from the 13th week of the first semester to the second week of the second semester of the academic year.

\section{Instruments}

In this research, we collected data using the SASE tool. The SASE was developed and validated by Keskin et al. (2020) in Turkish. It is a 46-item 7-point scale ranging from 1 (strongly disagree) to 7 (strongly agree). Higher score indicates higher social anxiety. Since the scale was developed in Turkish, the scale was adapted in the following stages. The forward-backward translation procedure was applied to translate the SASE from Turkish into English language. The final translation was done and revised independently by two translators, and minor terms were edited. All authors approved the final developed version. The scale focused on learner-learner and learner-instructor interactions subscales to measure the social anxiety levels. There were nine questions related to students' demographics and 23 items targeting the learner-learner interaction subscale (e.g. 'In e-learning, I think that my participation/writing will be evaluated negatively on discussion pages'), 23 items targeting the learner-instructor interaction subscale (e.g. 'In e-learning, I think that my participation/writing will be evaluated negatively when communicating with the instructor'). Since the SASE comprises 46 items in total, it was seen as necessary to reach at least five times as many students as the total number of items, and if possible, 10 times as many students. Accordingly, the minimum number of students to be included in the study was calculated to be 300 . The data was collected via google forms ${ }^{\circledR ;}$ a survey administration software included as a part of web-based applications offered by google $\AA$.

\section{Important factors}

A second goal of this study was to examine the factors affecting social anxiety levels in e-learning. Based on the literature (Asher \& Aderka, 2018; Wittchen \& Fehm, 2001; Rao et al., 2007), we were interested in age and gender as prominent factors. 
The interaction between gender and age in relation to the social anxiety in e-learning was also examined.

We did not examine the association between factors such as GPA, marital status, and social anxiety in e-learning for a few reasons. Since students had just started online learning, studying the association between social anxiety in e-learning and GPA might not be suitable as the effect on the GPA would not be visible yet. Marital status was not examined since most students were single. Year of study was seen as an interesting variable to include, but since it was found to be correlated with age, only age was included in the analysis. Nevertheless, cumulative GPA, year of study, and marital status were included in the survey to have a clear understanding of overall students' characteristic. Additionally, the association between the perception of overall academic performance during e- learning and social anxiety in e-learning was examined. Performance was assessed with one question 'Which of the following describes your academic performance during e-learning the most?' The response to the question was 'overall performance enhanced', 'overall performance decreased', and 'no change in overall performance'.

\section{Statistical tests}

Firstly, confirmatory factor analysis (CFA) and a factorial validity analysis was conducted to identify the item-factor relations and the relationships between the factors. To assess the construct validity of the scale, convergent and divergent validity tests were performed based on average explained variance (AVE) values (Fornell \& Larcker, 1981).

The bivariate associations between the variables were examined. Associations between the variables were tested using chi-square test. A two-way between-subjects multivariate analysis of variance (MANOVA) was carried out to determine the association between gender (male vs. female students), age ( $\leq 21$ and $>21$ years old) and interaction between gender and age and social anxiety in e-learning. Age was categorised in this manner because 21 was the median age of this sample. Before the multivariate analyses, assumptions of parametric statistics (i.e. normality, multicollinearity, homogeneity of variances, and linearity) were tested. When a main effect or an interaction was significant for scores on the two subscales in a multivariate test, a univariate ANOVA test was performed separately for each subscale to locate the sources of the differences.

Partial $\eta 2$ was used as an estimate of effect size for the MANOVA (partial $\eta 2$ : small $=0.01$, medium $=0.06$, and large $=0.14)($ Cohen, 1988). Cohen's $d$ was used to estimate the magnitude of difference between the comparative groups, where effect sizes of 0.8 or greater were considered large, those of 0.5 medium, and less than 0.2 small (Cohen, 1988). A multinomial logistic regression was used to examine the associations between performance in e-learning and social anxiety levels. Reference category was no change in overall performance. Collinearity was tested and variance inflation factor (VIF) was acceptable (VIF =1). A p-value less than 0.05 was considered statistically significant. Statistical analysis was performed using SPSS (IBM SPSS Statistics, v25; IBM Corp). 


\section{Results}

Results of this study are presented in two sub-sections. The first section evidences the scale's validation and reliability, and the second section examines students' social

Table 1 CFA of the alternative models

\begin{tabular}{llllllll}
\hline & Models & Sub-Scales & $\chi^{2}$ & RMSEA & NFI & NNFI & CFI \\
\hline Original & Three-factor & Learner-learner & 3.18 & 0.09 & 0.97 & 0.98 & 0.98 \\
& (correlated) & Learner-Instructor & 4.26 & 0.10 & 0.97 & 0.97 & 0.98 \\
Adaptation study & Three-factor & Learner-learner & 3.27 & 0.09 & 0.93 & 0.94 & 0.95 \\
& (uncorrelated) & Learner-Instructor & 3.03 & 0.08 & 0.96 & 0.97 & 0.97 \\
\hline
\end{tabular}

Abbreviations: CFA, confirmatory factor analysis; RMSEA, root mean square error of approximation; NFI, normed fit index; NNFI, non-normed fit index; CFI, comparative fit index

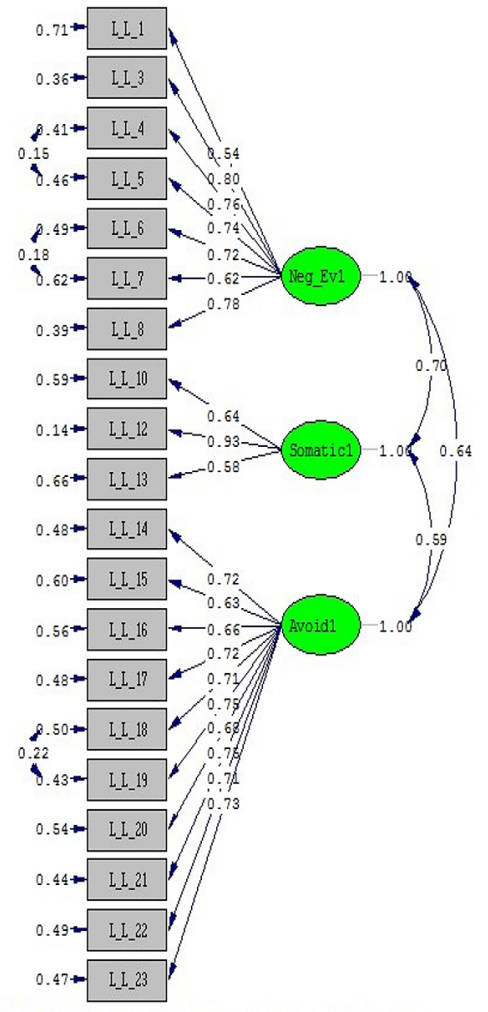

a

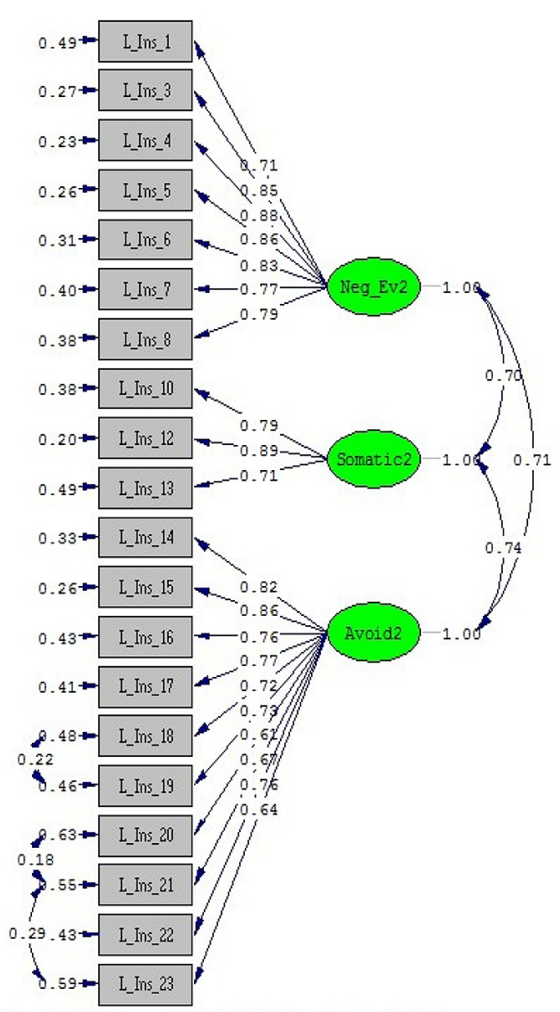

b

Fig. 1 Factor structure of the scale (standardised coefficients) in the adaptation study. a Factor structure of learner-learner interaction sub-scale, b Factor structure of learner-instructor interaction sub-scale 
anxiety in accordance with their gender, age, and perceived academic performance.

\subsection{Reliability and validity of the SASE}

In total, 325 students participated in the study, with an overall response rate of $41.6 \%$. Observations that distorted the data distribution and participants that did not fill the collection tool in accordance with the instruction were removed from the data set. Data of 306 students were used in the final data analysis. Six items with low construct validity were removed from the data collection tool.

\subsubsection{Confirmatory factor analysis and model comparison}

Table 1 presents three factor correlated model values of fit for original and English version. The results of alternative models here show that the three-factor model (i.e. negative evaluation, somatic symptoms, and avoidance of interaction), which was validated in the original scale development study, was thus confirmed. Figure 1 shows the factor-item parameters of the scale. According to the adaptation study, learner-learner interaction explained $61 \%$ of the total variance and learner-instructor interaction explained $67.96 \%$ of the total variance of the three-factor structure.

The standardised factor loads between the items of the scale and the factor structures showed that all factor loads were greater than 0.30 . Further, the factor loads were statistically significant; therefore, it can be stated that a total of 40 items in learner-learner interaction and learner-instructor interaction successfully measured the assumed substructures, and the scales' factorial validity is confirmed.

Table 2 AVE and reliability coefficients for the sub-scales

\begin{tabular}{|c|c|c|c|c|c|}
\hline & & Dimensions & AVE & $\begin{array}{l}\text { Structural Re- } \\
\text { liability }(\omega)\end{array}$ & $\begin{array}{l}\text { Cron- } \\
\text { bach's } \\
\text { Alfa } \\
(\alpha)\end{array}$ \\
\hline \multirow[t]{6}{*}{ Learner-learner } & \multirow[t]{3}{*}{ Original study } & $\mathrm{NE}$ & 0.67 & 0.95 & 0.95 \\
\hline & & SS & 0.74 & 0.92 & 0.92 \\
\hline & & AI & 0.66 & 0.95 & 0.95 \\
\hline & \multirow[t]{3}{*}{ Adaptation study } & $\mathrm{NE}$ & 0.51 & 0.88 & 0.88 \\
\hline & & SS & 0.54 & 0.77 & 0.77 \\
\hline & & AI & 0.50 & 0.91 & 0.91 \\
\hline \multirow[t]{6}{*}{ Learner-instructor } & \multirow[t]{3}{*}{ Original study } & $\mathrm{NE}$ & 0.77 & 0.97 & 0.97 \\
\hline & & SS & 0.76 & 0.93 & 0.93 \\
\hline & & AI & 0.74 & 0.97 & 0.97 \\
\hline & \multirow[t]{3}{*}{ Adaptation study } & $\mathrm{NE}$ & 0.66 & 0.93 & 0.93 \\
\hline & & SS & 0.64 & 0.84 & 0.85 \\
\hline & & AI & 0.54 & 0.92 & 0.93 \\
\hline
\end{tabular}

Abbreviation: NE, negative evaluation; SS, somatic symptoms; AI, avoidance of interaction 


\subsubsection{Construct validity}

The reliability coefficients are expected to be greater than 0.70 and AVE values are greater than 0.50 (Nunnally \& Bernstein, 1994). Moreover, AVE value must be less than the coefficient of construct validity. Table 2 presents the AVE values and reliability values of the scale

The AVE values for the sub-scale of learner-learner and learner-instructor interaction (Table 2) for all three factors were less than the structural reliability coefficients and greater than 0.50 . Further, the structural reliability and the Cronbach's Alfa $(\alpha)$ coefficients are expected to be greater than 0.70 . As seen in Table 2, the scale met the requirements for convergence and divergence validity

Table 3 shows the inter-factor correlation coefficients and the square root values of the AVE values for the sub-scales. To verify the discriminant validity of the scale, the square root of each construct's AVE must be higher than its correlation with another construct (Fornell \& Larcker, 1981). As seen in Table 3, the values met this criterion and thus the discriminant validity of the scale was established

\subsection{Examining social anxiety across gender and age}

\subsubsection{Students' characteristics}

Regarding students' characteristics, 189 (61.8\%) respondents were male, whereas $117(38.2 \%)$ respondents were female. Most students were aged 21 years or less $(\mathrm{N}=244,79.7 \%)$, while the rest $(\mathrm{N}=62,20.3 \%)$ were older than 21 years. Regarding marital status, $301(98.4 \%)$ students were single and five $(1.6 \%)$ were married. There were $152(49.7 \%)$ 3rd year medical students, 138 (45.1\%) 4th year students, while 5 th and 6th year accounted for minority (5.2\%). In total, 209 (68.3\%) respondents' GPA were excellent (4.75-5), whereas 61 (19.9\%) respondents' GPA were considered as very good (4.5-4.74), and $36(11.8 \%)$ respondents' GPA was good (below $<4.5)$. When students were asked to describe their performance during e-learning, $144(47.1 \%)$ agreed that their performance improved, while $73(23.9 \%)$ reported that their performance decreased, and 89 (29.1\%) did not find any change in their performance. Additionally, students in age group 21 years old or less had an excellent GPA of 5-4.75 compared to students in age group older than 21 years old $(71.7 \% \mathrm{vs}$. $54.8 \%$, chi-squared $=26.7, p<0.001)$. No differences were found between male and

Table 3 Discriminant validity measures

\begin{tabular}{llllllll}
\hline Learner-learner & & & & \multicolumn{3}{l}{ Learner-instructor } \\
\hline AI & SS & NE & Dimensions & NE & SS & AI \\
& & $\mathbf{0 . 7 1 *}$ & NE & $\mathbf{0 . 8 1 *}$ & & \\
& $\mathbf{0 . 7 3 *}$ & 0.70 & SS & 0.70 & $\mathbf{0 . 8 0}^{*}$ & \\
$\mathbf{0 . 7 1 *}$ & 0.59 & 0.64 & AI & 0.71 & 0.74 & $\mathbf{0 . 7 4 *}$ \\
\hline
\end{tabular}

* The diagonal elements of the matrices are the divergence validity coefficients, which were obtained through the calculation of the square root values of the AVE

Abbreviation: NE, negative evaluation; SS, somatic symptoms; AI, avoidance of interaction 
female students regarding GPA. No significant differences were found in academic performance between male and female students during e-learning $(p>0.05)$

\subsubsection{Descriptive statistics for SASE scores and relationship between the subscales}

The descriptive statistics reporting social anxiety in e-learning among the students are shown in Table 4: the SASE learner-learner (mean $=79.9, \mathrm{SD}=23.6$, range 21-135) and learner-instructor social anxiety (mean $=77.8, \mathrm{SD}=26$, range 22-138). Learnerlearner social anxiety was strongly correlated to SASE learner-instructor social anxiety $(r=0.8, p<0.001)$

\subsubsection{Gender, Age, and Interaction Effect}

Factor variances met the equality of variances assumption (Box's $M=13.3, F=1.45$, $P=0.161)$. The MANOVA results showed that gender had a significant effect on the combined subscales (Wilks' $\lambda=0.97, F(2,301)=4.15, P=0.02$, partial $\eta^{2}=0.03$ ). Table 4 presents the means and standard deviations of the groups

The univariate analysis ANOVA on each subscale score (Table 5) found significant difference between female and male students in the learner-learner social anxiety subscale, after controlling for participants' age $(F(1,302)=7.12, p=0.008$, partial $\left.\eta^{2}=0.03\right)$. The mean female students' learner-learner social anxiety score $(\mathrm{M}=86.3, \mathrm{SD}=24.6)$ was statistically significantly higher than that of the male stu-

Table 4 Mean (standard deviation) of students' social anxiety in e-learning by gender and age

Table 5 ANOVA results: Test of gender, age, and interaction effect $(n=306)$

\begin{tabular}{llllll}
\hline & $\begin{array}{l}\text { All } \\
\text { students } \\
(\mathrm{n}=306)\end{array}$ & $\begin{array}{l}\text { Male } \\
\text { students } \\
(\mathrm{n}=189)\end{array}$ & $\begin{array}{l}\text { Female } \\
\text { students } \\
(\mathrm{n}=117)\end{array}$ & $\begin{array}{l}\text { Age }<=21 \\
(\mathrm{n}=244)\end{array}$ & $\begin{array}{l}\text { Age }>21 \\
(\mathrm{n}=62)\end{array}$ \\
\hline $\begin{array}{l}\text { Learn- } \\
\text { er- }\end{array}$ & $79.9(23.6)$ & $75.9(22.2)$ & $86.3(24.6)$ & $80.3(23.5)$ & $78.2(24.3)$ \\
$\begin{array}{l}\text { learner } \\
\begin{array}{l}\text { Learn- } \\
\text { er- in- } \\
\text { structor }\end{array}\end{array}$ & & & & \\
\hline
\end{tabular}

\begin{tabular}{llllll}
\hline Effect & $\begin{array}{l}\text { Social anxiety } \\
\text { subscales }\end{array}$ & $d f$ & $F$ & $P$ & $\begin{array}{l}\text { Par- } \\
\text { tial } \\
\eta^{2}\end{array}$ \\
\hline Gender & Learner- learner & 1,302 & 7.1 & 0.008 & 0.03 \\
& Learner- instructor & 1,302 & 3.09 & 0.08 & 0.01 \\
Age & Learner- learner & 1,302 & 0.65 & 0.42 & 0.002 \\
& Learner- instructor & 1,302 & 6.22 & 0.013 & 0.025 \\
Gender * age & Learner- learner & 1,302 & 0.42 & 0.51 & 0.001 \\
& Learner- instructor & 1,302 & 0.43 & 0.50 & 0.001 \\
Error & Learner- learner & 302 & & & \\
& Learner- instructor & 302 & & & \\
\hline
\end{tabular}


dents $(\mathrm{M}=75.9, \mathrm{SD}=22.2)$. This difference has a relatively medium effect (Cohen's $\mathrm{d}=0.44)$

The interaction between gender and learner-instructor social anxiety was not significant $(p=0.08)$. Female students' learner-instructor social anxiety $(\mathrm{M}=82.8$, $\mathrm{SD}=26.1)$ was higher than the learner-instructor social anxiety for male students $(\mathrm{M}=74.7, \mathrm{SD}=25.6)$, but the difference was not statistically significant

A significant main effect for age group was found (Wilks' $\lambda=0.96, F(2,301)=6.08$, $P=0.003$, partial $\eta^{2}=0.04$ ), indicating significant differences between the levels of age group on the subscales. The univariate analysis on each subscale score found significant difference between students younger than 21 years of age and those older than 21 years of age in the learner-instructor social anxiety subscale $(F(1,302)=6.22$, $p=0.013$, partial $\left.\eta^{2}=0.025\right)$. Students younger than 21 years of age had significantly higher learner-instructor social anxiety $(\mathrm{M}=79.6, \mathrm{SD}=25.8)$ compared to students older than 21 years of age $(\mathrm{M}=70.9, \mathrm{SD}=25.9)$. The overall effect was small to medium difference between age groups (Cohen's $d=0.33$ ). No statistically significant differences were found between age group in relation to the learner-learner social anxiety. Analyses did not detect a statistically significant gender by age group interaction effect (Wilks' $\lambda=0.99, F(2,301)=0.23, P=0.79$, Partial $\eta^{2}=0.002$ )

\subsubsection{Perception of performance in e-learning and SASE}

In learner-learner interaction, the mean score of the students responded with a decrease in the academic performance $(\mathrm{M}=83.6, \mathrm{SD}=20.4)$ was higher than those who reported no change $(\mathrm{M}=82.1, \mathrm{SD}=24.20)$, and students who noticed an improved academic performance $(\mathrm{M}=76.6, \mathrm{SD}=24.5)$. Similarly, in learner-instructor interaction, the highest mean score was for those who recorded a decrease in the academic performance $(\mathrm{M}=81.9, \mathrm{SD}=22.9)$, while the least mean score was for students who observed an improved academic performance $(\mathrm{M}=75.2, \mathrm{SD}=27.5)$. In addition, the mean score of students that reported no notable change in academic performance was $(\mathrm{M}=78.6, \mathrm{SD}=25.9)$

Results of multinomial regression analysis show that the model was not statistically significant $\left(\chi^{2}=6.4, p=0.17\right)$, indicating that although social anxiety levels were higher in students with decreased perceived performance during e-learning compared to students with enhanced performance, the levels of social anxiety could not significantly differentiate between the perception of students' performance

\section{Discussion}

The purpose of this study was to assess medical students' social anxiety levels in e-learning environments. We tested the reliability and validity of the English version of the SASE and compared social anxiety levels by gender and age groups. Despite existing research assessing social anxiety within the educational context, this study is the first to examine the impact of the shift towards e-learning on social anxiety and associated socio-demographic factors among medical students. The findings provided evidence of the SASE's reliability and validity based on factor analysis and 
suggested that in e-learning environments, medical students' social anxiety significantly differed according to gender and age

The English version of the SASE contains a total of 40 items: 20 for learner-learner interaction and 20 for learner-instructor interaction. The present results can facilitate wider use of the instrument as a general measure of social anxiety in e-learning. The validity and reliability assessments of the subscales were conducted separately. This allows researchers to use the subscales together or separately in e-learning environments

Social anxiety levels were significantly higher among female students than male students in learner-learner interaction. In learner-instructor interaction, there was no such statistically significant difference, indicating that both genders interact similarly with course instructors. The general cultural expectation that women should be modest, quiet, and demure could potentially justify these results. A recent study among Saudi medical students found that social anxiety levels in men were higher than those in women (Elhadad et al., 2017). Other studies have demonstrated no statistically significant gender difference (Al-Hazmi et al., 2020; Hakami et al., 2018). An American study (Asher \& Adreka, 2018) measuring social anxiety reported higher levels among women than men. Additionally, women were more likely to be distressed, worried, emotionally upset, and exhibit severe clinical symptoms. Besides the paucity of research examining social anxiety, there is an overt discrepancy in the available data about gender differences and their correlations with social anxiety. Hence, future studies examining these interconnections are required

The results revealed a statistically significant effect for age with respect to social anxiety in learner-instructor interaction. Older students reported lower anxiety as compared to those 21 years or younger. However, in learner-learner interaction, age had no significant effect on social anxiety. A possible explanation is that as students advance in their academics, they become more aware about how to interact with instructors, leading to progressive improvements in their social skills. The onset of social anxiety is in early to mid-adolescence, and the older a person gets, the less likely they will be to experience social anxiety (Wittchen \& Fehm, 2001). Future research should examine other variables that may affect the nature of the relationship between social anxiety in learner-instructor interaction and age. For example, students' expectations from the course, whether student have met the teacher face-toface before, and the instructor's gender and reputation may play a role in the level of social anxiety. Other factors potentially linked to the e-learning environment, such as the technical infrastructure in the student's learning environment, and computer self-efficacy (Chiu \& Wang, 2008), could also affect the level of social anxiety in e-learning

We found no significant association between social anxiety levels and perceived academic performance. One plausible explanation is that the study was conducted in the middle of the first semester of e-learning. Hence, while there may have been an association, it might not yet have become evident. In addition, as most students who responded to the survey (around 80\%) had a very good to excellent GPA, the significance of the association may have been affected. No previous studies have examined the impact of social anxiety levels on academic performance during e-learning. However, multiple studies have assessed the relationship between the two elements within 
the context of traditional teaching. A Canadian study found a significant negative impact of social anxiety on academic performance (Brook \& Willoughby, 2015), and a local study reported a significant negative correlation between social anxiety levels and academic performance (Al-Hazmi et al., 2020). Although this topic is discussed frequently in the context of traditional learning, there is a need for more research to investigate social anxiety in e-learning and its impact on academic performance

\section{Limitations}

The current study did not compare social anxiety in normal classes and e-learning as no previous data were available on the levels of social anxiety among the sampled medical students. Additionally, previous studies have found an association between social anxiety and other comorbidities that affect both the physical and mental aspects, such as substance dependence, post-traumatic stress disorder, depression disorder, and cardiovascular diseases (Leichsenring \& Leweke, 2017; Hakami et al., 2018). This highlights the need for more studies exploring the occurrence of any unpleasant comorbidities to reduce their burden and improve quality of life. Moreover, while our study aimed to evaluate social anxiety in e-learning among undergraduate medical students, as the setting was a single university, the results have limited generalisability. While medical training is probably universally rigorous, cultural traits within educational settings most likely differ across countries. Furthermore, the current study did not incorporate all different variables that might interfere with students' social anxiety levels, such as the students' learning environment, lack of face-face interactions with the course instructor, and the nature of the course (e.g. theoretical or applied). Finally, the study used a self-report approach to collect data, which could have introduced response bias (Althubaiti, 2016)

\section{Conclusion}

No research was conducted on social anxiety in e-learning environment, hence, there appears to be a need to introduce the concept to individuals in the field of education. Future research on the implications of social anxiety in education are needed. Studies that discuss the role of gender, age, and academic performance especially in the context of e-learning. Further, the use of SASE may signal the start of a new line of social anxiety research that focuses on virtual environment. The observed differences in social anxiety levels among students of different age and gender, provide evidence to teachers and leaders in medical education to integrate strategies that will lessen the anxiety of students and hopefully lead to better educational and clinical environments

Acknowledgements None.

Funding None. 


\section{Declarations}

Conflict of interest No competing interest to report.

Ethics approval and consent to participate Students were informed (using consent forms and cover letter) about the aim of the study and were ensured anonymity and confidentiality. In addition, they were assured that participation would not affect their academic progress. Participation in the study was voluntary during the semester. Data were kept confidential and were used only for the study's aim. After the ethical review, the study was approved by the Institutional Review Board at the King Abdullah International Medical Research Centre.

\section{References}

Al-Hazmi, B. H., Sabur, S. S., \& Al-Hazmi, R. H. (2020). Social anxiety disorder in medical students at Taibah University, Saudi Arabia. Journal Family Medicine and Primary Care Review, 9(8), 43294332. https://doi.org/10.4103/jfmpc.jfmpc_915 20

Althubaiti, A. (2016). Information bias in health research: definition, pitfalls, and adjustment methods. Journal of multidisciplinary healthcare, 4(9), 211-217. doi: https://doi.org/10.2147/JMDH.S104807

Asher, M., \& Aderka, I. M. (2018). Gender differences in social anxiety disorder. Journal of Clinical Psychology, 74(10), 1730-1741. https://doi.org/10.1002/jclp.22624

Bandelow, B., \& Michaelis, S. (2015). Epidemiology of anxiety disorders in the 21 st century. Dialogues in clinical neuroscience, 17(3), 327-335. https://doi.org/10.31887/DCNS.2015.17.3/bbandelow

Brook, C. A., \& Willoughby, T. (2015). The social ties that bind: social anxiety and academic achievement across the university years. Journal of Youth and Adolescence, 44(5), 1139-1152. https://doi. org/10.1007/s10964-015-0262-8

Chiu, C., \& Wang, E. T. (2008). Understanding Web-based learning continuance intention: The role of subjective task value. Information management journal, 45, 194-201

COVID-19 Educational Disruption and Response. UNESCO (2020, March 24). https://en.unesco.org/ news/covid-19-educational-disruption-and-response

Cox, B. J., Fleet, C., \& Stein, M. B. (2004). Self-criticism and social phobia in the US national comorbidity survey. Journal of Affective Disorders, 82(2), 227-234. https://doi.org/10.1016/j.jad.2003.12.012

Elhadad, A. A., Alzaala, M. A., Alghamdi, R. S., Asiri, S. A., Algarni, A. A., \& Elthabet, M. M. (2017). Social phobia among Saudi medical students. Middle East Current Psychiatry, 24(2), 68-71

Fornell, C., \& Larcker, D. F. (1981). Structural Equation Models with Unobservable Variables and Measurement Error: Algebra and Statistics. International journal of market research, 18(3), 382-388. https://doi.org/10.1177/002224378101800313

Hakami, R. M., Mahfouz, M. S., Adawi, A. M., Mahha, A. J., Athathi, A. J., Daghreeri, H. H. ... Areeshi, N. A. (2018). Social anxiety disorder and its impact in undergraduate students at Jazan University, Saudi Arabia. Mental Illness, 9(2), 7274. https://doi.org/10.4081/mi.2017.7274

Leichsenring, F., \& Leweke, F. (2017). Social Anxiety Disorder. The New England Journal of Medicine, 376(23), 2255-2264. https://doi.org/10.1056/NEJMcp1614701

Nunnally, J. C., \& Bernstein, I. H. (1994). Psychometric theory (3rd ed., pp. 248-292p). New York: McGraw-Hill

Rao, P. A., Beidel, D. C., Turner, S. M., Ammerman, R. T., Crosby, L. E., \& Sallee, F. R. (2007). Social anxiety disorder in childhood and adolescence: descriptive psychopathology. Behaviour Research and Therapy, 45(6), 1181-1191. https://doi.org/10.1016/j.brat.2006.07.015

Reta, Y., Ayalew, M., Yeneabat, T., \& Bedaso, A. (2020). Social Anxiety Disorder Among Undergraduate Students of Hawassa University, College of Medicine and Health Sciences, Ethiopia. Neuropsychiatric Disease and Treatment, 16, 571-577. https://doi.org/10.2147/NDT.S235416

Keskin, S., Şahin, M., Uluç, S., \& Yurdugul, H. (2020). Online learners' interactions and social anxiety: the social anxiety scale for e-learning environments (SASE). Interactive learning environments. doi: https://doi.org/10.1080/10494820.2020.1769681 
Neufeld, C. B., Palma, P. C., Caetano, K., Brust-Renck, P. G., Curtiss, J., \& Hofmann, S. G. (2020). A randomized clinical trial of group and individual Cognitive-Behavioral Therapy approaches for Social Anxiety Disorder. International journal of clinical and health psychology: IJCHP, 20(1), 29-37. https://doi.org/10.1016/j.ijchp.2019.11.004

Stein, M. B., \& Stein, D. J. (2008). Social anxiety disorder. Lancet, 371(9618), 1115-1125. https://doi. org/10.1016/S0140-6736(08)60488-2

Van Ameringen, M., Mancini, C., \& Farvolden, P. (2003). The impact of anxiety disorders on educational achievement. Journal of anxiety disorders, 17(5), 561-571. https://doi.org/10.1016/ s0887-6185(02)00228-1

Vaona, A., Banzi, R., Kwag, K. H., Rigon, G., Cereda, D., Pecoraro, V. .. Moja, L. (2018). E-learning for health professionals. The Cochrane Database of Systematic Reviews, 1(1), CD011736. https://doi. org/10.1002/14651858.CD011736.pub2

Wittchen, H. U., \& Fehm, L. (2001). Epidemiology, patterns of comorbidity, and associated disabilities of social phobia. Psychiatric Clinics of North America, 24(4), 617-641. https://doi.org/10.1016/ s0193-953x(05)70254-9

Publisher's Note Springer Nature remains neutral with regard to jurisdictional claims in published maps and institutional affiliations.

\section{Authors and Affiliations}

\section{Ali S Alsudais ${ }^{1,2}$. Abdullah S Alghamdi, ${ }^{1,2}$ Abdullrhman A Alharbi, ${ }^{1,2}$. Atif A Alshehri ${ }^{1,2} \cdot$ Mustafa A Alzhrani ${ }^{1,2} \cdot$ Sinan Keskin ${ }^{3} \cdot$ Muhittin Şahin ${ }^{4}$. Alaa M Althubaiti ${ }^{1,2,5}$}

Alaa M Althubaiti

thubaitia@ksau-hs.edu.sa

1 College of Medicine, King Saud bin Abdulaziz University for Health Sciences, Jeddah, Saudi Arabia

2 King Abdullah International Medical Research Centre, Jeddah, Saudi Arabia

3 Computer Education and Instructional Technology, Van Yuzuncu Yil University, Van, Turkey

4 Computer Education and Instructional Technology, Ege University, Izmir, Turkey

5 College of Medicine, King Saud bin Abdulaziz University for Health Sciences, Mail Code 6656, P.O. Box 9515, 21423 Jeddah, Saudi Arabia 